\title{
SUCCESS-DRIVING BUSINESS MODEL CHARACTERISTICS OF IAAS PROVIDERS - TOWARDS A QUANTITATIVE EVALUATION
}

\author{
Sebastian Floerecke, Florian Felix Röck and Franz Lehner \\ Chair of Information Systems (Information and IT Service Management), \\ University of Passau, Passau, Germany
}

\begin{abstract}
Despite the highly competitive situation within the Infrastructure as a Service (IaaS) market and the resulting pressure and uncertainty for the involved providers, only little knowledge is available about business model characteristics (BMCs) related to success. Merely few qualitative studies are existing that propose hypotheses on success-driving business model characteristics (SDBMCs), however, a general and comparative quantitative evaluation and thus an evidence for their impact on business success is still missing. But this knowledge is essential for IaaS providers as it would allow them to focus their limited resources and efforts on the truly decisive BMCs and, at the same time, save costs by avoiding activities and investments of minor importance. Aiming to reduce this gap, a web-based survey was carried out, in which representatives of IaaS providers of different size rated the level of relevance of the proposed SDBMCs. As this study is still going on, this paper focuses on presenting the study design and an analysis of the data collected so far. As a preliminary result, nearly $80 \%$ of the SDBMCs were rated as extremely important or important, meaning that the existing qualitative research results were confirmed to a high degree. The relevance of the individual SDBMCs varies greatly depending on the IaaS provider's size.
\end{abstract}

\section{KEYWORDS}

Infrastructure as a Service (IaaS), Business Model, Success-Driving Business Model Characteristics (SDBMCs), Web-Based Survey, Quantitative Evaluation, Behavioural-Science Research Paradigm.

\section{INTRODUCTION}

Infrastructure as a Service (IaaS), which primarily stands for providing basic infrastructural resources (compute, storage and network) [1], is the fastest-growing cloud computing market segment worldwide. Gartner [2] forecasts a market volume of approximately 62 billion USD in 2021, a doubling compared to 2018. About three quarters of the IaaS market is dominated by four globally acting, so-called hyperscalers: Alibaba, Amazon Web Services, Google and Microsoft [3]. The hyperscalers are particularly characterized by a high level of standardization, a low price, a high and global availability, a broad and integrated cloud service portfolio and a self-service sale [4]. The remaining ongoing declining market share is divided up between several large international but also national IT companies and a great number of smaller providers focusing on regional markets [5]. Due to the high growth rate of the IaaS market, also the non-hyperscalers have been able to gain annual market volume over the years. However, it will probably become problematic for this group, when a market saturation is reached and the hyperscalers increasingly address their current key customer segments [6]. An absolute prerequisite for the long-term success of non-hyperscalers is a business model that differs from the hyperscalers and thus, the pursuit of a differentiation strategy. To compete directly with the hyperscalers is a hopeless endeavour [7]. The major reason for the growing market power of the hyperscalers is the smooth transformation of basic IaaS services (IaaS services without extensions such as managed or platform services) into a commodity [8]. Commodities are products and 
services that are highly standardized and to a large extent equivalent regarding functionality and quality, independently of a specific vendor [9]. The main characteristic of commodity markets, including, e.g., the electricity and gas market, are the high importance of economies of scale and thus the company size. Only this way the increasing pricing pressure can be countered [7].

Despite the highly competitive situation within the IaaS market and the associated pressure and uncertainty among the involved providers, research offers only little guidance on what business model characteristics (BMCs) shape success $[4,5,10]$. So far, only two studies propose hypotheses on IaaS-specific success-driving business model characteristics (SDBMCs) based on explorative multiple case-studies with 21 cloud providers of various sizes [7,8] and 18 small and medium-sized regional IaaS providers, respectively [6]. A general and comparative quantitative evaluation of these hypotheses according to the behavioural-science research paradigm [11] is, however, still pending. Therefore, an evidence for their impact on business success has not yet been provided. But this knowledge is essential for IaaS providers as it would allow them to focus their limited resources and efforts on the truly decisive BMCs and, at the same time, save costs by omitting investments in minor important aspects. Aiming to reduce this research gap, this paper addresses two following research questions (RQs):

\section{RQ1: What business model characteristics of IaaS providers have the highest impact on success?}

\section{RQ2: To what extent do company size-specific differences exist in this regard?}

In order to answer both RQs, a first round of a web-based survey was carried out, in which high-level representatives of IaaS providers assessed the level of relevance of the proposed SDBMCs on a five-point Likert scale. The arithmetic mean values for all respondents and for specific company sizes (small, medium and large) reflect the perceived impact on success. Overall, this study represents the second phase in a mixed-method approach [12] consisting of (1) the generation (qualitative research) and (2) the validation (quantitative research) of hypotheses [11] with the intention of developing a theory for explaining Gregor [13]. The study is still running, so this paper presents the study design but also first results based on the data captured so far.

\section{BACKGROUND AND RELATED WORK}

Cloud computing has become an enabler for new, innovative business models both on the provider's and customer's side [1]. A business model is a system comprising a set of interrelated components or partial models for depicting, implementing, innovating and evaluating the business logic of a company [14]. This construct builds upon central theories, including the resource-based view, the strategic network theory and the transaction cost theory, and is mostly described as an intermediary between a company's strategy and its business processes [15]. Business models have especially played an important role in explaining differences in company success: the same technology may result in different economic outcome, depending on the way it is exploited and marketed by a business model [16]. Although there is no consensus on the specific set of components forming a business model, a comprehensive and widespread business model framework is the Business Model Canvas [17]. Its components comprise value propositions, key resources, key activities, partner network, customer segments, channels, customer relationships, revenue streams and cost structure [17]. Each component can exhibit various design options called business model characteristics (BMCs) [8].

The current state of research on success-driving business model characteristics (SDBMCs) of IaaS providers in general, but in particular of different types and sizes is dissatisfying $[4,5]$. Overall, several scholars (e.g., $[10,18]$ ) have ignored that the cloud computing ecosystem entails a multitude of companies that offer a variety of products and services, such as IaaS, Platform as a Service (PaaS) and Software as a Service (SaaS), and additionally act, e.g., as aggregator, consultant, integrator or market place operator and thus may considerably differ from 
one another [19]. Undifferentiated, ecosystem role-independent investigations of business models hence provide only a low level of explanatory power [8]. In the case of IaaS providers, where the focus is on operating huge server farms and achieving economies of scale - and not on providing software or advisory activities - it is of central importance to further differentiate in terms of company size [5]. Available research on IaaS-specific SDBMCs is limited to recent qualitative studies by Floerecke and Lehner: Based on two exploratory multiple case-studies with 21 cloud providers of various sizes [7, 8] and 18 small and medium-sized regional IaaS providers [6], they derived several hypotheses on SDBMCs (see section 3 and 4 for details). A general and comparative quantitative evaluation, however, is still pending and therefore conducted in this study.

\section{RESEARCH DESIGN}

\subsection{Research Method}

The overall intention of this study is to generate a theory for explaining. Theories for explaining elucidate primarily how and why a specific phenomenon occurs in a certain situation [13]. To reach the research goal, the behavioural-science research paradigm is applied, whose objective is to (1) discover and (2) justify causal, explanatory and/or predictive relations for information systems phenomena. Such phenomena typically are located in the area of conflict between individuals or organizations, tasks and technologies [11]. Whereas the hypotheses on SDBMCs by Floerecke and Lehner [6-8] can be assigned to the discovery phase, the quantitative evaluation is part of the justification phase. In other words, this study is the second step of a mixed-method approach combining, by definition, a qualitative and a quantitative research method [12]. To conduct the quantitative evaluation, a web-based survey with high-ranked representatives of IaaS providers was chosen. A web-based survey combines a cost- and time-efficient approach with a high outreach [20]. This data collection instrument has a long and important tradition in verifying SDBMCs and success factors (e.g., [21, 22]).

\subsection{Survey Design}

The existing hypotheses on SDBMCs of IaaS providers were consolidated and expanded by further prospective BMCs, derived by the long-term experience of the authors in the cloud computing domain. The resulting list of BMCs ( $\mathrm{C} 1$ to $\mathrm{C} 36$ in figure 1$)$, sorted by the business model components, represented the footing of the survey.

To capture the perceived impact of the individual BMCs on company success, the participants had to rate them on a five-point Likert scale (1: not important, 2: slightly important, 3: fairly important, 4: important, 5: extremely important). This approach follows the relevant literature from other domains (e.g., [22, 23]). In order not to force the participants to answer each question and therefore, to avoid possible distortions, the response category "no opinion" was offered [24]. They had to assess only those BMCs that were part of their own current business model, otherwise, the specific BMC was skipped. In addition, further information on the companies' background, such as foundation year, size and turnover, was gathered.

The survey was launched in German and English using SoSciSurvey, a free survey tool for academic purposes. A pre-test with practitioners was omitted because the questionnaire was adjusted to already well-established question structures and scales (cf., [21, 22, 25]). Nevertheless, two scholars checked the survey for its intelligibility and consistency, which resulted in slight refinements.

\subsection{Data Collection}

The population consisted of providers offering IaaS services. As no comprehensive list of IaaS providers exists, an own database was created. To this end, the Google search engine was used 
to identify IaaS providers and provider lists maintained by market research institutes or practitioners' journals. In addition, Crunchbase, a platform offering relevant business information, and an internal database developed and managed by the research institute were utilized. By this means, 560 companies were identified in total. For 55 companies no contact information could be found. The final dataset therefore consisted of 505 IaaS providers stemming from 50 countries. USA (181), Germany (178) and Great Britain (44) are the mostly represented countries.

Initially, the survey was distributed by e-mail using the official info address or contact form on the providers' websites. This request contained information on the study's background and goal as well as the weblink to the survey. The desired group of participants were employees holding a managerial position and being responsible or at least co-responsible for the business model. Only one person per company was intended to participate. To reduce nonresponse bias, all companies were guaranteed anonymity and two reminders were sent out [20]. Beside the e-mail request, the study was promoted using two business-oriented social networking sites (LinkedIn and Xing) and by a well-known German IT journal (IT-Business). The first survey period comprised six weeks.

Within this period, the survey website was visited 215 times. However, only 24 (19 from Germany) data records were usable. The 24 companies are spread evenly across company sizes (small, medium and large - EU Recommendation 2003/361/EC). Not considered were 175 participants who did not answer any of the questions and further 16 participants who only partially completed the survey. The required time for filling out the survey was about 26 minutes on average. Based on the 505 companies being part of the created database, the response rate was $4.75 \%$. This low value is in line with similar studies in other domains (e.g., [21, 23, 26]).

\subsection{Data Analysis}

IBM SPSS Statistics was used to analyse the data. Three sub-groups were created, one for each company size (small, medium and large). For each BMC, the arithmetic mean, minimum, maximum and standard deviation of the answers were calculated. The arithmetic mean reflects the perceived impact of an individual BMC on success [22,23]. On this basis, a ranking of all SDBMCs was compiled. In accordance with similar studies from other domains [21], all hypotheses on SDBMCs with a mean of 3.5 and higher were accepted. SDBMCs with an average value equal or superior to 4.5 (marked in bold in figure 1) were classified as highly relevant for business success because they are within the top ten percent range of the scale. A BMC with a mean below 3.5 (marked in italics in figure 1) is considered as a factor that does not contribute to business success substantially, whereby the underlying hypothesis was rejected.

\section{RESULTS}

Figure 1 shows the mean values of the BMCs, ordered by the various business model components. It entails the results for small, medium-sized and large IaaS providers, followed by all respondents ("All"). "n/a" means that a BMC was assessed by nobody or only one person. Overall, the participants rated nearly $80 \%$ of the proposed SDBMCs as extremely important or important and thereby confirmed the existing qualitative research results to a considerable extent. The BMCs with the highest average ratings over all respondents (threshold value of 4.3 because of low number of items with 4.5 and higher) are highly qualified personnel, personal sales process, strict service level agreements (SLAs), transition and integration services, managed services, extensive customer support, high user experience, functioning referral marketing and both after sales service and cross-selling with a personal point of contact. These top-rated BMCs refer to various business model components and can be partly classified as cross-domain and IaaS-specific. Unexpectedly, out of the $17 \mathrm{BMCs}$, which were additionally added to the set of hypotheses by the authors, 11 were considered as relevant $(>=3.5)$. 
International Journal on Cloud Computing: Services and Architecture (IJCCSA) Vol. 10, No. 4/5, October 2020

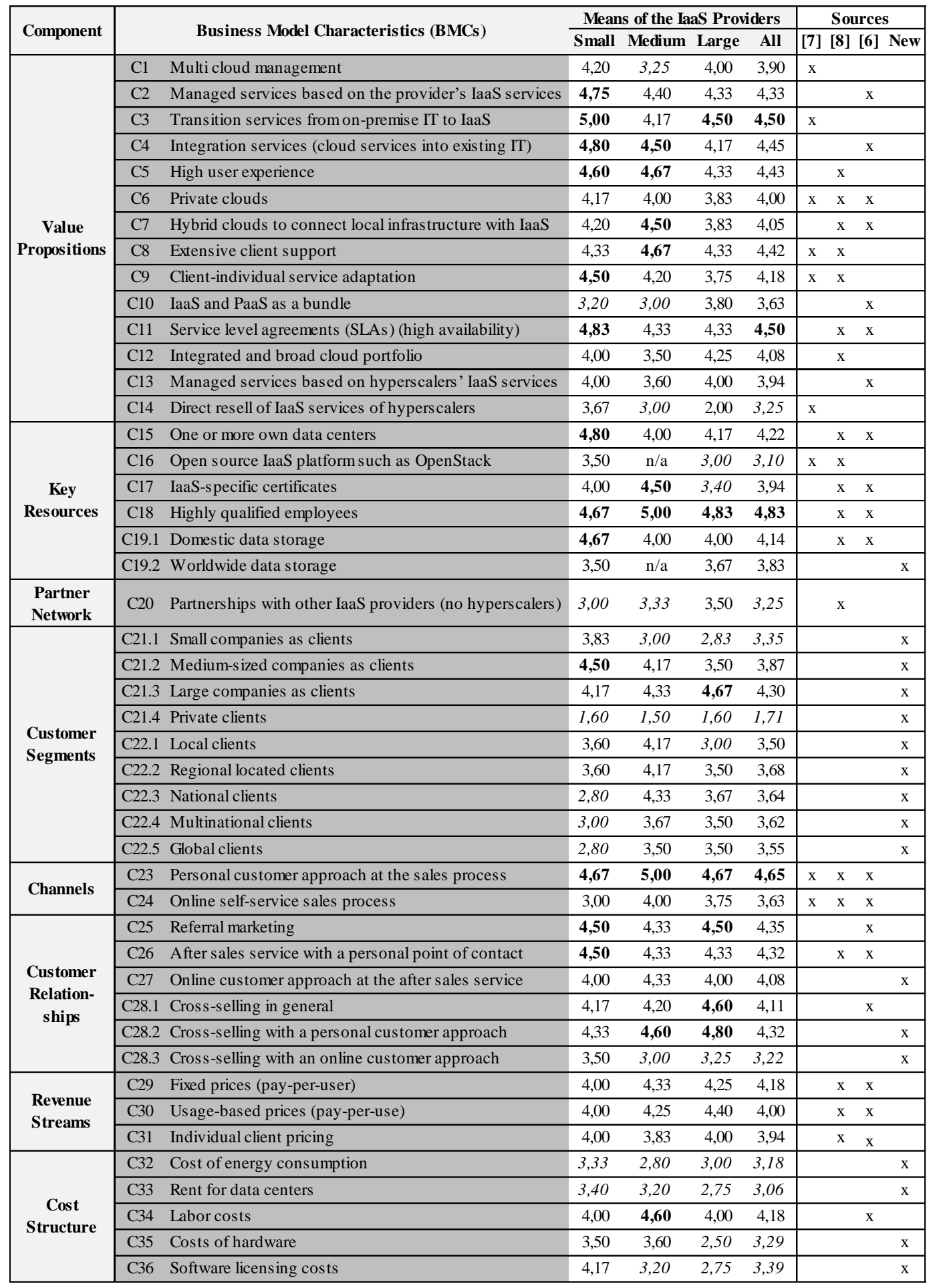

Figure 1. Top ten ranked company size-specific and -overarching SDBMCs

\section{The results concerning rejected BMCs (All) can be summarized as follows:}

- As there are numerous providers focusing on unmodified reselling of hyperscalers' IaaS services [6], it is surprising that the cooperation among IaaS providers in general and reselling of hyperscalers' IaaS services in particular was rated low. This could stem from fearing cannibalization of the own IaaS offerings. To share resources and customers on a large scale (cloud federation) in order to achieve greater economies of scale [27] thus received only little support. 
- A similarly low average value got the usage of open source IaaS platforms, such as OpenStack and Eucalyptus. Contrary to the prevailing assumption [7], most customers do not seem to be deterred by a proprietary IaaS platform and an associated vendor lock-in effect.

- The online channel is compared to the personal communication seen as clearly less effective concerning cross-selling activities. Customers seem to appreciate a personal contact.

- Private customers play a less important role within the IaaS business. The main interest of this customer group is focused primarily on ready-to-use applications (SaaS). If they obtain IaaS services, they mostly choose one of the hyperscalers [5].

- Concerning the cost structure, rental and electricity costs for data centres as well as hardware and software licensing costs are not relevant for success. In line with the assumption [6], only in the area of personnel costs a decisive optimization and savings potential is seen.

\section{The company-size specific analysis showed the following results:}

- Small IaaS providers additionally consider customer-specific adaptation of IaaS services, own data centres and domestic data storage most important. This might be because small IaaS providers have no option, but to occupy a niche of the IaaS market by establishing a close, personal relationship and offering an individual response to customer wishes and needs. Their main customers prefer a provider within their region that guarantees domestic data storage and particularly no data exchange with US (Cloud Act) and Chinese (China Internet Security Law) data centres [5].

- Medium-sized IaaS providers find hybrid cloud scenarios and IaaS-specific certifications as decisive. The reason for this might be that they mainly address medium-sized and large companies within their country that commonly operate numerous legacy on-premise systems requiring a high sensitivity regarding compliance [6]. For both small and mediumsized IaaS providers, offering PaaS in addition to IaaS is not regarded as a promising option. There seems to be a lack of resources and skills needed for a successful development of a PaaS platform in order to compete with the innovativeness and speed of the large providers and particularly the hyperscalers [8].

- Large IaaS providers highlight cross-selling in general (particularly with a personal customer approach) in combination with an integrated and broad cloud portfolio. Both aspects are mutually reinforcing each other. In addition, by means of a broad portfolio, a provider has full control over all aspects of the service provision and can therefore offer higher security and service quality [8]. Apart from that, large IaaS providers prefer primarily large companies as customers, irrespectively of their location. One cause for this might be companies' higher available financial resources for cloud services on the one hand, and the providers' technical abilities on the other hand [6]. Surprisingly, large IaaS providers perceive the ownership of IaaS-specific certificates compared to smaller providers as relatively unimportant. An explanation could be that certificates are considered as standard and thus as basic precondition with increasing provider size.

Figure 2 presents a condensed overview of the ten most important SDBMCs. Those SDBMCs being in the top ten of all provider sizes are marked in grey.

\begin{tabular}{|c|c|c|c|c|c|c|c|c|}
\hline Rank & \multicolumn{2}{|r|}{ Small } & \multicolumn{2}{|r|}{ Medium } & \multicolumn{2}{|r|}{ Large } & \multicolumn{2}{|r|}{ All } \\
\hline 1 & $\mathrm{C} 3$ & Transition services & $\mathrm{C} 18$ & Qualified employees & $\mathrm{C} 18$ & Qualified employees & $\mathrm{C} 18$ & Qualified employees \\
\hline 2 & C11 & SLAs & $\mathrm{C} 23$ & Personal sales process & $\mathrm{C} 28.2$ & 2 Personal cross-selling & $\mathrm{C} 23$ & Personal sales process \\
\hline 3 & $\mathrm{C} 4$ & Integration services & $\mathrm{C} 5$ & High user experience & $\mathrm{C} 23$ & Personal sales process & $\mathrm{C} 3$ & Transition services \\
\hline 4 & C15 & Own data centers & $\mathrm{C} 8$ & Extensive client support & $\mathrm{C} 21.3$ & 3 Large firms as clients & C11 & SLAs \\
\hline 5 & & Managed services & $\mathrm{C} 28$. & 2 Personal cross-selling & C28.1 & 1 Cross-selling in general & $\mathrm{C} 4$ & Integration services \\
\hline 6 & C19.1 & Domestic data storage & $\mathrm{C} 34$ & Labor costs & $\mathrm{C} 25$ & Referral marketing & $\mathrm{C} 5$ & High user experience \\
\hline 7 & C18 & Qualified employees & $\mathrm{C} 4$ & Integration services & & Transition services & $\mathrm{C} 8$ & Extensive client support \\
\hline 8 & $\mathrm{C} 23$ & Personal sales process & $\mathrm{C} 7$ & Hybrid clouds & $\mathrm{C} 30$ & Usage-based prices & $\mathrm{C} 25$ & Referral marketing \\
\hline 9 & $\mathrm{C} 5$ & High user experience & $\mathrm{C} 17$ & IaaS-specific certificates & $\mathrm{C} 8$ & Extensive client support & $\mathrm{C} 2$ & Managed services \\
\hline 10 & $\mathrm{C} 25$ & Referral marketing & $\mathrm{C} 2$ & Managed services & $\mathrm{C} 2$ & Managed services & $\mathrm{C} 26$ & Personal after sales ser \\
\hline
\end{tabular}

Figure 2. Top ten ranked company size-specific and -overarching SDBMCs 
The importance of managed services, integration services, private cloud, customer-specific adaptation, direct resell of hyperscalers' IaaS services, rent for datacentres and costs for software licenses as well as fixed prices decrease with rising provider size. This is in line with the fact that both small and medium-sized IaaS providers must differentiate themselves by additional services as they cannot compete with their large competitors in the commodity part. Regarding pricing models, no substantial differences on fixed, usage-based and individual client pricing could be found. Obviously, IaaS providers of all sizes are expected to offer multiple pricing models, where the customers can choose one according to their specific needs. Mediumsized IaaS providers assess labour costs disproportionally with a high impact on success. The reasons for this might be the focus of small IaaS providers on few projects with a high demand for customization that offset the costs incurred and, on the contrary, the high standardization and automation of large IaaS providers leading to lower employee requirement. Medium-sized IaaS providers are somehow stuck in the middle. Therefore, they must take care that their offering is not perceived as a commodity that is badly positioned in terms of price. To conclude, the differences between the three provider sizes mainly originate from the specific requirements of the various addressed customer segments: The importance of small and medium-sized customers decreases, and the importance of large customers increases with rising provider size.

\section{CONCLUSION}

\subsection{Limitations}

As this study is still going on, this paper presented the study design and preliminary results based on the data captured so far. The sample size of this first round of data collection is relatively small. Hence, especially the company size-specific results must be regarded as a first indication. The plan is to continue with the data collection aiming to increase the sample size substantially and thus, to provide more conclusive results leading to a theory for explaining at the end. A larger sample size will allow to apply sophisticated statistical methods, such as a factor analysis and a t-test. The companies being part of the generated database will be contacted again via e-mail and in the case of no response via telephone.

Beside the small sample size and a high proportion of Germany-based IaaS providers, the study underlies the general weakness that the answers of the participants reflect their personal experience and opinion. Even if this corresponds with the common approach in the scientific community (e.g., $[21,25]$ ) and so-called perceived SDBMCs (particularly by senior managers) are widely consistent with the factual ones (e.g., [28, 29]), possible distortions cannot be ruled out. Apart from that, Likert scales are prone to the central tendency bias - the tendency to place items in the middle of the scale [30]. To reduce this influence, the questions were formulated as clearly and unambiguously as possible and a no-opinion response option was offered [24]. Finally, it remains unknown to what extent the participants were really in line with the required profile.

\subsection{Contributions and Future Work}

Despite the relatively early stage of this research, there are already notable contributions. Basically, this is the first quantitative study on SDBMCs of IaaS providers. The existing qualitative research results could be confirmed to a high degree. Beyond that, further BMCs could be identified as relevant, whereby the literature basis was expanded. A further contribution lies in the promising reference for future empirical investigations of business models of various cloud ecosystem roles (cf., [19]). As a practical contribution, IaaS providers of different sizes obtain a source for their business model creation and innovation process, allowing them to focus their limited resources and efforts on the truly decisive BMCs and, at the same time, save costs by omitting investments in minor important aspects. A weak performance in one or more of the top-rated BMCs can lead to a deterioration of the competitive situation. However, addressing all 
of them is not a guarantee for success. A business model can only lead to success when it is supported by adequate business processes and managed properly [15].

To conclude, the authors hope that they can expand the relatively low level of research on SDBMCs of IaaS providers and contribute to a higher awareness of the importance of this research topic in view of the increasing disappearance of smaller IaaS providers, the augmented withdrawal of also large providers of the (public) IaaS segment and ultimately, the growing market concentration among the hyperscalers.

\section{REFERENCES}

[1] Marston, S., Li, Z., Bandyopadhyay, S., Zhang, J., and Ghalsasi, A. (2011), "Cloud Computing The Business Perspective," Decision Support Systems, Vol. 51, No. 1, pp. 176-189.

[2] Statista (2019), "Revenues from Public Cloud Infrastructure as a Service (IaaS) Market Worldwide from 2015 to 2017, by Vendor (in Million U.S. Dollars)," Statista, Hamburg, Germany.

[3] Costello, K. and Goasduff, L. (2019), "Gartner Says Worldwide IaaS Public Cloud Services Market Grew 31.3\% in 2018," Gartner, Stamford, USA.

[4] Floerecke, S., Ertl, C., and Herzfeldt, A. (2020), "Major Drivers for the Rising Dominance of the Hyperscalers in the Infrastructure as a Service Market Segment," International Journal of Cloud Computing. (In Press)

[5] Floerecke, S. and Lehner, F. (2019), "Dominant Business Model Patterns of Regional IaaS Providers - An Exploratory Multiple-Case Study," presented at the 16. International Conference on Economics of Grids, Clouds, Systems, and Services, Leeds, England.

[6] Floerecke, S. and Lehner, F. (2019), "Der ungleiche Kampf um Marktanteile im Infrastructureas-a-Service-Segment: Erfolgswirksame Geschäftsmodellcharakteristika regionaler Anbieter im Wettbewerb mit den Hyperscalern," HMD - Praxis der Wirtschaftsinformatik, Vol. 56, No. 6, pp. 1334-1354.

[7] Floerecke, S. and Lehner, F. (2018), "Business Model Characteristics for Local IaaS Providers for Counteracting the Dominance of the Hyperscalers," presented at the 15. International Conference on Economics of Grids, Clouds, Systems, and Services, Pisa, Italy.

[8] Floerecke, S. and Lehner, F. (2018), "Success-Driving Business Model Characteristics of IaaS and PaaS Providers," International Journal on Cloud Computing: Services and Architecture (IJCCSA), Vol. 8, No. 6, pp. 1-22.

[9] Bruhn, M. (2011), "Commodities im Dienstleistungsbereich," in Commodity Marketing, M. Enke and A. Geigenmüller, Eds., 1. ed., Wiesbaden: Gabler, pp. 57-77.

[10] Labes, S., Hanner, N., and Zarnekow, R. (2017), "Successfull Business Model Types of Cloud Providers," Business \& Information Systems Engineering, Vol. 59, No. 4, pp. 223-233.

[11] March, S. T. and Smith, G. F. (1995), "Design and Natural Science Research on Information Technology," Decision Support Systems, Vol. 15, No. 4, pp. 251-266.

[12] Kaplan, B. and Duchon, D. (1988), "Combining Qualitative and Quantitative Methods in Information Systems Research: A Case Study," Management Information Systems Quarterly, Vol. 12, No. 4, pp. 571-586.

[13] Gregor, S. (2006), "The Nature of Theory in Information Systems," Management Information Systems Quarterly, Vol. 30, No. 3, pp. 611-642.

[14] Veit, D. et al. (2014), "Business Models - An Information Systems Research Agenda," Business \& Information Systems Engineering, Vol. 56, No. 1, pp. 55-64.

[15] Wirtz, B., Pistoia, A., Ullrich, S., and Göttel, V. (2016), "Business Models: Origin, Development and Future Research Perspectives," Long Range Planning, Vol. 49, No. 1, pp. 36-54. 
[16] Lambert, S. C. and Davidson, R. A. (2013), "Applications of the Business Model in Studies of Enterprise Success, Innovation and Classification: An Analysis of Empirical Research from 1996 to 2010," European Management Journal, Vol. 31, No. 6, pp. 668-681.

[17] Osterwalder, A. and Pigneur, Y., Business Model Generation (2010), A Handbook for Visionaries, Game Changers, and Challengers, 1. ed., New Jersey: John Wiley \& Sons.

[18] Trenz, M., Huntgeburth, J., and Veit, D. (2019), "How to Succeed with Cloud Services?," Business \& Information Systems Engineering, Vol. 61, No. 2, pp. 181-194.

[19] Floerecke, S., Lehner, F., and Schweikl, S. (2020), "Cloud Computing Ecosystem Model: Evaluation and Role Clusters," Electronic Markets. DOI: 10.1007/s12525-020-00419-2.

[20] Evans, J. R. and Mathur, A. (2005), "The Value of Online Surveys," Internet Research, Vol. 15, No. 2, pp. 195-219.

[21] Nah, F. F. H., Zuckweiler, K. M., and Lee-Shang Lau, J. (2003), "ERP Implementation: Chief Information Officers' Perceptions of Critical Success Factors," International Journal of HumanComputer Interaction, Vol. 16, No. 1, pp. 5-22.

[22] Yusof, S. R. M. and Aspinwall, E. M. (2000), "Critical Success Factors in Small and Medium Enterprises: Survey Results," Total Quality Management, Vol. 11, No. 4-6, pp. 448-462.

[23] Arthur Jr, W. and Bennett Jr, W. (1995), "The International Assignee: The Relative Importance of Factors Perceived to Contribute to Success," Personnel Psychology, Vol. 48, No. 1, pp. 99114.

[24] Schnell, R., Hill, P. B., and Esser, E. (2018), Methoden der empirischen Sozialforschung, 11. ed., Berlin, Boston: De Gruyter Oldenbourg.

[25] Durst, M. and Daum, M. (2007), "Erfolgsfaktoren serviceorientierter Architekturen," HMD Praxis der Wirtschaftsinformatik, Vol. 44, No. 1, pp. 18-27.

[26] Ramli, H. S. B., Chin, A. L. L., and Choo, A. C. P. (2016), "Career Success for Women in Higher Education Institution: The Factors Influencing the Success of Women Academician," International Business Management, Vol. 10, No. 17, pp. 3929-3935.

[27] Kim, K., Kang, S., and Altmann, J. (2014), "Cloud Goliath Versus a Federation of Cloud Davids," presented at the 11. International Conference on Economics of Clouds, Grids, Systems and Services, Cardiff, UK.

[28] Leidecker, J. K. and Bruno, A. V. (1984), "Identifying and Using Critical Success Factors," Long Range Planning, Vol. 17, No. 1, pp. 23-32.

[29] Grunert, K. G. and Sørensen, E. (1996), "Perceived and Actual Key Success Factors: A Study of the Yoghurt Market in Denmark," Aarhus School of Business, MAPP Centre, Aarhus, Denmark.

[30] Foddy, W. (1994), Constructing Questions for Interviews and Questionnaires: Theory and Practice in Social Research, Cambridge: Cambridge University Press. 


\section{AUTHORS}

Dr. Sebastian Floerecke is working as IT Solution Manager at Munich International Airport. Before that, he was research associate and doctoral candidate at the Chair of Information Systems (Information and IT Service Management) of University of Passau. In his dissertation project, he researched the business ecosystem and success-driving business model characteristics in the field of cloud computing. His research has been published in international journals, including Electronic Markets and International Journal of Service Science, Management, Engineering, and Technology, and conferences, such as European Conference on Information Systems and Internationale Tagung Wirtschaftsinformatik.

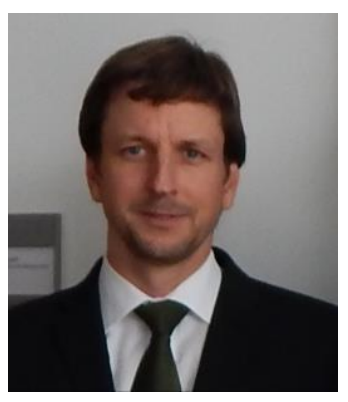

Florian Felix Röck, M.Sc. is currently working as Real Estate Investment Analyst at Woehr + Bauer in Munich. Prior to that, he graduated at the Chair of Information Systems (Information and IT Service Management) of the University of Passau including a semester abroad at the Craig School of Business at California State University. In his master thesis, he dealt with the evaluation of success-driving business model characteristics of IaaS providers of various sizes. He also holds a bachelor's degree as industrial engineer with focus on process engineering from Management Center Innsbruck.

Prof. Dr. Franz Lehner is a full professor (Chair of Information and IT Service Management) at the University of Passau. His academic career started at the Koblenz School of Corporate Management (WHU, Germany) with a chair in the field of information management. After a short period as founding president of the Danube-University at Krems, which is a centre for postgraduate studies in Austria, he became a full professor for Information Systems in 1996 at the University of Regensburg. In 2004 he accepted a call to the University of Passau. His research is focusing on information, IT and knowledge management.
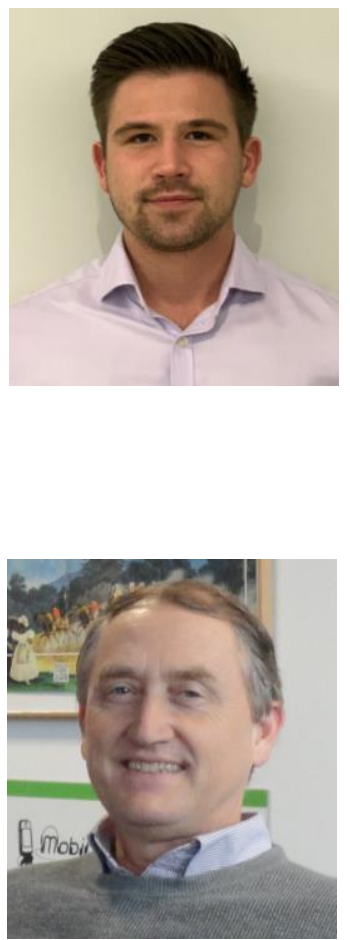